Revue d'histoire de l'Amérique française

REYUE D.HISTOIRE DE L'AMÉRIQUE FRANÇAISE

\title{
Communauté urbaine de Montréal, Les résidences. Montréal, Communauté urbaine de Montréal, 2 Complexe Desjardins, C. P. 129, Montréal, Qué. H5B 1E6, 1987. 803 p. 15,00 \$
}

\section{David B. Hanna}

Volume 41, numéro 4, printemps 1988

URI : https://id.erudit.org/iderudit/304625ar

DOI : https://doi.org/10.7202/304625ar

Aller au sommaire du numéro

Éditeur(s)

Institut d'histoire de l'Amérique française

ISSN

0035-2357 (imprimé)

1492-1383 (numérique)

Découvrir la revue

Citer ce compte rendu

Hanna, D. B. (1988). Compte rendu de [Communauté urbaine de Montréal, Les résidences. Montréal, Communauté urbaine de Montréal, 2 Complexe

Desjardins, C. P. 129, Montréal, Qué. H5B 1E6, 1987. 803 p. 15,00 \$1. Revue

d'histoire de l'Amérique française, 41(4), 605-607.

https://doi.org/10.7202/304625ar 
COMMUNAUTÉ URBAINE DE MONTRÉAL, Les résidences. Montréal, Communauté urbaine de Montréal, 2 Complexe Desjardins, C. P. 129, Montréal, Qué. H5B 1E6, 1987. 803 p. 15,00\$

C'est en 1980 que le premier cahier du répertoire d'architecture traditionnelle sur le territoire de la Communauté urbaine de Montréal paraît. En tout onze cahiers ont été publiés dont Les résidences est le plus récent et certainement le plus volumineux. Avec seulement trois derniers cahiers à paraitre, la série tire à sa fin. Toutefois, ayant traité de l'architecture religieuse, civile, militaire, industrielle, commerciale et rurale, cette dernière tranche portant essentiellement sur l'architecture domestique s'avère la plus difficile à rédiger de par sa complexité et ses proportions quantitatives.

L'espace résidentiel est le plus vaste des espaces fonctionnels de la zone urbanisée. Il est aussi le plus varié puisqu'un très grand nombre d'architectes, de contracteurs, de constructeurs et d'individus l'ont créé. Les critères de sélection des témoins de ce patrimoine domestique sont particulièrement importants puisque l'espace résidentiel, plus que toute autre espace, représente notre vécu individuel et collectif, nos valeurs et nos modes de vie, notre culture et notre société. Finalement ces critères et les bâtiments retenus au répertoire traduisent clairement ce que la CUM projette de faire quant à la protection de son patrimoine immobilier puisqu'elle entend contrôler la démolition des bâtiments répertoriés.

L'architecture urbaine domestique répertoriée pour fins de protection selon le schéma d'aménagement paraîtra dans trois volumes: Les résidences; Immeubles d'habitation; Maisons en rangée. Nous apprenons dans l'introduction de Les résidences que le titre équivaut à «Hôtels particuliers». Effectivement, des 303 maisons paraissant dans ce volume, $71 \%$ sont indubitablement des hôtels particuliers, c'est-à-dire de grandes maisons détachées, $23 \%$ sont des maisons attachées à d'autres, mais passablement somptueuses et conçues individuellement, puis $6 \%$ sont des maisons jumelées du style «Château Dufresne» ou «Maison Shaughnessy», donc des maisons exceptionnelles.

Il s'agit clairement d'un répertoire de l'architecture domestique bourgeoise de l'île de Montréal avant 1940. À ce titre, l'ouvrage est fort bien fait et les choix sont assez judicieux, même si l'on pourra toujours disputer l'exclusion d'un hôtel favori. Si l'on prend le temps de repérer spatialement les bâtiments retenus, nous avons une carte assez fidèle de l'espace bourgeois montréalais. Le fameux anneau bourgeois autour du Mont-Royal prédomine avec $86 \%$ des entrées au répertoire. Fait non surprenant, Westmount et le Mille-Carré, le flanc anglais de la montagne, l'emportent sur le flanc français, c'est-à-dire Outremont et le Plateau, en descendant vers le Carré Viger. Voilà le gros du livre, mais il reste une deuxième zone d'importance figurant au répertoire: le West Island riverain avec $8 \%$ des cas retenus. Cette zone se définit comme le «Lakeshore» du lac Saint-Louis, Senneville, et la frange longeant la rivière des Prairies aux environs de Sainte-Geneviève, Pierrefonds et Saraguay. Le Vieux Montréal, gîte original de la bourgeoisie montréalaise, compte pour $4 \%$ des choix du répertoire, ce qui apparaît normal quand on se rappelle la quasi-disparition de ce secteur au profit d'un nouveau centre-ville commercial au 19e siècle. 
Avec $98 \%$ des choix retenus, la zone bourgeoise s'arrête là à toutes fins pratiques. Si Notre-Dame-de-Grâce ou Ahuntsic se croyaient bourgeois, la réponse serait «pas suffisamment» puisqu'ils sont à peine touchés. Tout l'est de l'île à partir de l'avenue De Lorimier offre quatre maisons au répertoire et on devine tout de suite que deux sont au Château Dufresne.

Les aspects techniques du répertoire sont impressionnants. Au moins une photo récente illustre chaque bâtiment. Celle-ci est toujours claire, ensoleillée et suffisamment grande pour permettre de bien scruter l'architecture. Parfois on y ajoute une deuxième photo pour témoigner d'une étape intermédiaire avant la restauration, et même une troisième pour montrer l'état d'origine. Pour les hôtels les plus somptueux, il y a aussi des illustrations de détail et d'intérieur. Toutes les photos sont datées. Souvent un schéma situe le bâtiment pour qu'il n'y ait aucune confusion, et pour localiser les remises et dépendances.

Voilà pour la documentation visuelle. Du côté archivistique, le plus grand bijou s'avère une liste des étapes de construction (y compris les annexes et remises). Grâce à un dépouillement des contrats notariés de construction, des permis municipaux de construction, des revues de construction et d'architecture de l'époque et d'autres sources, nous avons une liste très complète des architectes et entrepreneurs impliqués dans la construction, la modification et le réaménagement des bâtiments avec les dates et des renvois infrapaginaux systématiques. On ne peut que rester fortement impressionné par l'immense travail qui a été fait et la fiabilité des résultats de recherche. En plus de ces informations archivistiques, un court texte nous renseigne sur les anciens occupants de ces maisons.

L'ouvrage est bien organisé, facile à consulter et bien indexé. Les maisons sont présentées par ordre alphabétique des premiers occupants, mais on trouve à la fin un index chronologique dans lequel apparaissent aussi les architectes. On soupçonne que le classement aurait été beaucoup plus utile s'il eût été organisé par ordre spatial (rues et quartiers) ou par ordre chronologique. Il est intéressant de constater que $2 \%$ des hôtels de ce répertoire ont été bâtis au $18 \mathrm{e}$ siècle, $6 \%$ durant la première moitié du $19 \mathrm{e}$, et $34 \%$ durant la deuxième. La part du lion (58\%) revient aux années 1900-1936. Curieusement, malgré la date limite de 1939 du répertoire, le dernier bâtiment répertorié date de 1936.

Il reste à faire une critique de fond qui est importante compte tenu de l'incidence directe du répertoire sur le plan d'aménagement de la CUM et sur la conservation patrimoniale. Où figurent les maisons populaires dans ce répertoire? Il n'y a aucune maison dans Les résidences qui se vendrait pour moins d'un quart de million de dollars aujourd'hui, et même celles-là sont rares puisque la forte majorité valent un million et plus. Le prochain volume à paraître, soit Immeubles d'habitation, ne portera-t-il que sur cette forme d'habitation collective bourgeoise qui se répand à Montréal à partir de 1900? Le troisième volume, Maisons en rangée, laisse aussi supposer un penchant nettement plus bourgeois puisque les entrepreneurs fabriquaient les projets domiciliaires en rangée, souvent avec l'aide d'un architecte, pour le marché de luxe. Les maisons populaires étaient généralement faites à la pièce, puis accollées les unes aux autres. Nulle part d'ailleurs ne fait-on mention d'un volume sur les «plex», cette typologie résidentielle fabuleuse qui domine l'espace résidentiel de l'époque 1850-1939 et fait l'originalité de Montréal. 
Peut-être est-ce l'approche ponctuelle qui ne fonctionne plus au niveau des maisons populaires. Quelle maison choisir pour représenter sa classe? Pourtant Jean-Claude Marsan, dans son ouvrage Montréal en évolution, a clairement identifié, dès 1974, le phénomène de la «rue spectacle» (Marsan, p. 289292). Ainsi l'ensemble de quadruplex des années 1870 sur la rue Wolfe, construit chacun par un entrepreneur différent, forme un ensemble extraordinaire que Marsan cite et qui mérite d'être reconnu officiellement. C'est une perte sans égal que la Ville elle-même ait forcé depuis la démolition de cette rue. Il est aussi regrettable que d'autres rues semblables disparaissent sous l'impulsion de programmes de rénovation mal conçus qui permettent aux propriétaires d'arracher briques, linteaux, corniches, fenêtres et lucarnes et de les remplacer par des façades banales et insipides. Des rues entières sont dévalorisées à jamais.

Pour conclure, il me semble que même dans son approche ponctuelle, la CUM commet une erreur très grave en ne considérant pas «les résidences» moins somptueuses mais tout aussi significatives pour le patrimoine domiciliaire. Que fait-on par exemple de la toute petite maison unifamiliale aux murs de crépi, sise au 1295 rue Montcalm au sud de Sainte-Catherine, dernier témoin du logement artisanal typique de l'est de Montréal, de l'époque de la grande conflagration de 1852, avant l'invasion des «plex»? Il en est de même de ce rare survivant unifamilial de Griffintown, sis au 175 rue de la Montagne, près de Wellington, et qui date des années 1840. Dans une autre époque et un autre lieu, pourquoi les résidences unifamiliales ouvrières construites en 1916, à Pointe-aux-Trembles, sur la 6e avenue, par le philanthrope Rosaire Prieur, ne méritent-elles pas d'être citées? Les exemples se multiplient très vite et le besoin est urgent.

Tout ceci ne dévalue pas un ouvrage qui représente une recherche extrêmement bien faite et qui reste un document bien organisé, un outil de référence précieux pour la CUM, les chercheurs et le public.

Département de géographie 